\title{
Optimization by Genetic Algorithm in Wireless Sensor Networks Utilizing Multiple Sinks
}

\author{
SHAUBAN ALI SOLANGI*, DIL NAWAZ HAKRO*, MUHAMMAD MEMON**, \\ KHALIL-UR-REHMAN KHOUMBATI*, AKHTAR HUSSAIN JALBANI***
}

RECEIVED ON 07.06.2018 ACCEPTED ON 30.10.2018

\begin{abstract}
WSN (Wireless Sensor Network) comprises of small-sized and constraint-capability SN (Sensor Nodes) which record, send and receive data, sensed to a sink. The network lifetime and energy usability are important challenges to be dealt with. During the working of the SN, the maximum amount of energy is consumed than sensing and processing of data. Therefore, an efficient transmission of the data is required so that the energy can be saved.

In this paper, a novel routing and scheduling method for WSNs using GA(Genetic Algorithm) is presented, where the sinks employed on four sides of the sensor field. These sinks collect the data from the SNs having the optimal distance towards the respective sink. The proposed scheme finds the optimized path using GA, during transmission of data from SN to the nearest sink. First, we run the GA for determination of routing paths, where a source SN finds the possible number of optimal hops. Second, the hops or intermediate relay SNs are assumed to relay the data towards the sink, efficiently.

The performance is experimented and evaluated using MATLAB R2016b. The simulations have carried out through comparing the proposed scheme with TEEN (Threshold Sensitive Energy Efficient Sensor Network Protocol). The results of simulation comprise of 10 and 20 number of SNs, discretely. Additionally, the direct distance of each node is calculated and the distance through multiple hops from/to the nearest sink is also evaluated. The achievements of the proposed technique are to save both energy and distance for the sake of network longevity and optimal and precise data delivery by multiple hops.
\end{abstract}

Key Words: Wireless Sensor Network, Genetic Algorithm, Network Lifetime, Routing, Optimization.

\section{INTRODUCTION}

$\mathrm{H}$ undreds of small motes construct a network via a radio connectivity. These motes are small electronic devices called as sensors.

Traditionally, the mote or sensor technology is the result of the progression in MEMS (Micro-Electro-MechanicalSystems). The MEMS technology is the pioneer of the development of the cheap and inexpensive small-sized sensors motes, which are driven by the supply of small

Authors E-Mail: (shauban@scholars.usindh.edu.pk, shauban76@gmail.com, dilnawaz@usindh.edu.pk,khalil.khoumbati@usindh.edu.pk, muhammadmemon90@gmail.com jalbaniakhtar@gmail.com)

* Institute of Information \& Communication Technology, University of Sindh, Jamshoro, Pakistan.

** Institute of Business Administration, University of Sindh, Jamshoro, Pakistan.

*** Department of Information Technology, Quaid-e-Awam University of Engineering, Science \& Technology, Nawabshah, Pakistan.

This is an open access article published by Mehran University Research Journal of Engineering and Technology, Jamshoro under the CC by 4.0 International License. 
scale battery. The batteries of the SNs are usually with limited amount of energy. Additionally, these batteries are irreplaceable due to large number of sensors and inaccessible environment. Due to small size of the SNs, main constraints such as limited bandwidth, processing and computational limitations and small-sized battery are imposed on the sensors. The interconnection of the variable and densely deployment of the SNs constructs a WSN. The SNs can be deployed in an environment in one of the two ways such as systematically or ad hoc. The deterministic or orderly deployment of the nodes is done in systematically distribution, whereas the ad hoc deployment of the SNs deals with the random placement of the SNs [1-2]. Applications of the WSNs encompasses a wide range of monitoring and tracking. Monitoring plays a pivotal role in environment monitoring, patient monitoring remotely, animal and habitat monitoring.
Furthermore, tracking comprises border surveillance, enemy tracking, and vehicle tracking.

The main components of the WSNs are SNs, sink, user or database administrator. The sink is called BS (Base Station). Where, the SNs generates data through sensing the vicinity of the sensors and transmit it towards the sink. The transmission of the generated data totally depends upon the energy of the battery. Having the smallsized battery the SN gets depleted in a very short time [34]. The energy exhaustion of the nodes is greater in transmission than sensing and computation. Therefore, it is essential to route the data effectively and efficiently for the sake of the lifetime of the sensor nodes.

The sensors route the data through direct communication paradigm and multiple hops communication methods as illustrated in Fig. 1(a-c).

(b) Direct Communication

(a) Multiple Hops

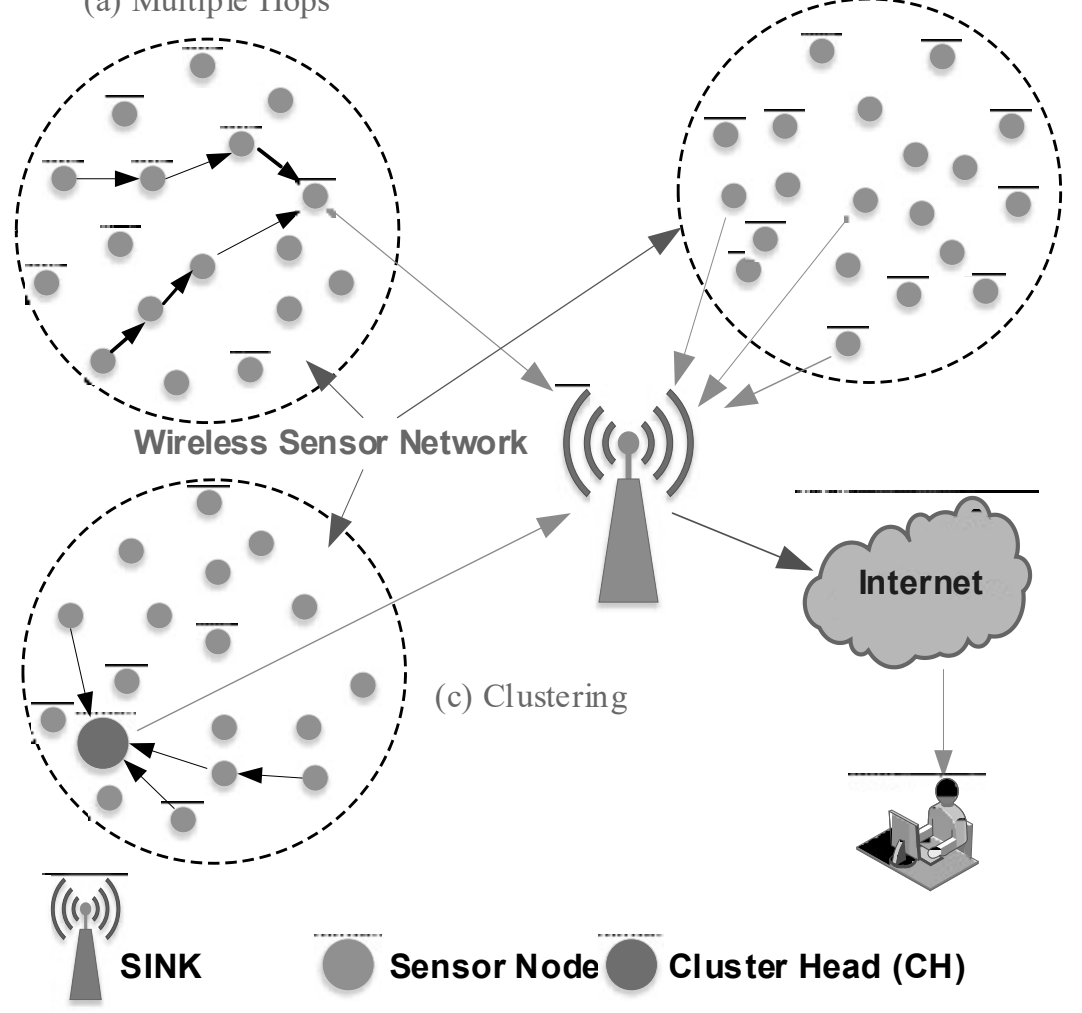

FIG. 1(a). MULTIPLE HOPS, (b) DIRECT COMMUNICATION AND (c) CLUSTERING SCHEMES 
Whereas, in Fig. 1(a) the trivial energy is consumed during the working of the sensors because each node routes the data towards the sinks is known as multiple hops communication method. In Fig. 1(b) the nodes transmit the data directly towards the sink, which is good for the nearer nodes but it is fatal for the farther SNs. This is called direct communication method [5]. Therefore, in Fig. 1(c) all sensor are bound to transmit the data to the cluster, which is called $\mathrm{CH}$ (Cluster Head). However, the optimal routing scheme is needed for the minimum energy consumption and effective routing of the data. The optimization in routing of the transmission of the data benefits with the trivial energy consumption.

Since last three decades, research scholars have been working and suggesting the research for resolving energy consumption issue. Different scholars have proposed different routing schemes for energy consumption. Optimization is one of the most efficient scheme for routing the data towards the sink by the optimized routes which saves the energy. The energy exhaustion is the key component of the sensor nodes which determines the lifespan of both the sensor nodes and WSNs.

\section{OVERVIEW OF GA}

GA is the meta-heuristic evolutionary algorithm, which mimic, the natural selection process. The GA is utilized, mostly, for the optimization, where it finds the most optimal solution from the available solutions [6-8]. The development of the GA leads by an American professor John Holland, University of Michigan, 1975. The GA dose the functional process of the selection of the best solution/individual with the help of its operators as shown in Fig. 2. The operators of GA have been taken with modifications. Specifically, the fitness function of the GA defines the fitness level of all individuals of the population. All of the nodes or chromosomes have been considered as one population [9-12]. Furthermore, the operation of the operators such as selection, fitness function, crossover and mutation is performed after the generation of the population of the fit chromosomes. The fit chromosomes of the population are assumed to be competent individuals for the further process of the selection of the fit solution.

We adopted general GA with some modifications for our research. The process commences with the selection of better individuals. These individuals are selected upon their energy level/efficiency. Additionally, the strongest individual are preferably selected leaving the weakest individuals. By this we get a papulation containing the strongest individuals. Then the selection process is applied as shown in Fig. 2. Every individual is supposed to undergone a selection process called binary tournament selection. This process gives the diverse number of optimal solutions. Then the crossover operator is invoked for the selection of best individual/solution from the current diverse number of solutions. Nevertheless, the crossover strategy is applied with certain fraction which constitutes route information of the other half of the other gene and vice versa. It is interesting to note that the selection and crossover method is applied for the sake of obtaining most feasible and diverse solutions and fittest number of genes as shown in Fig. 2. The combination of selected population with is subjected to the mutation $\xi$ where certain mutation probability is applied for obtaining the best one solution from the diverse number of solutions. After mutation the loops are removed and fitness of the new population is examined once more before proceeding to the future generation as illustrated in Fig. 2.

Mehran University Research Journal of Engineering \& Technology, Volume 38, No. 4, October, 2019 [p-ISSN: 0254-7821, e-ISSN: 2413-7219] 
In the following sections general terms of GA with their meaning are briefly discussed:

Population: Population or chromosome is established by considering all nodes of the WSNs as one. The all individual SNs are assumed to be the possible solutions with equal number and length. The length is in the form of strings. Furthermore, every solution or nodes is called genes of alleles in the GA terminology [3,10-11].
Selection: The selection operator selects all individuals corresponding to their fitness values. Each individual has a certain fitness value. The selected genes are supposed to go to the next generation with robust and higher level of energy.

Crossover: The crossover function implies for the generation of the fit individuals which is the result of the survival of two individuals as shown in Fig. 3. These two

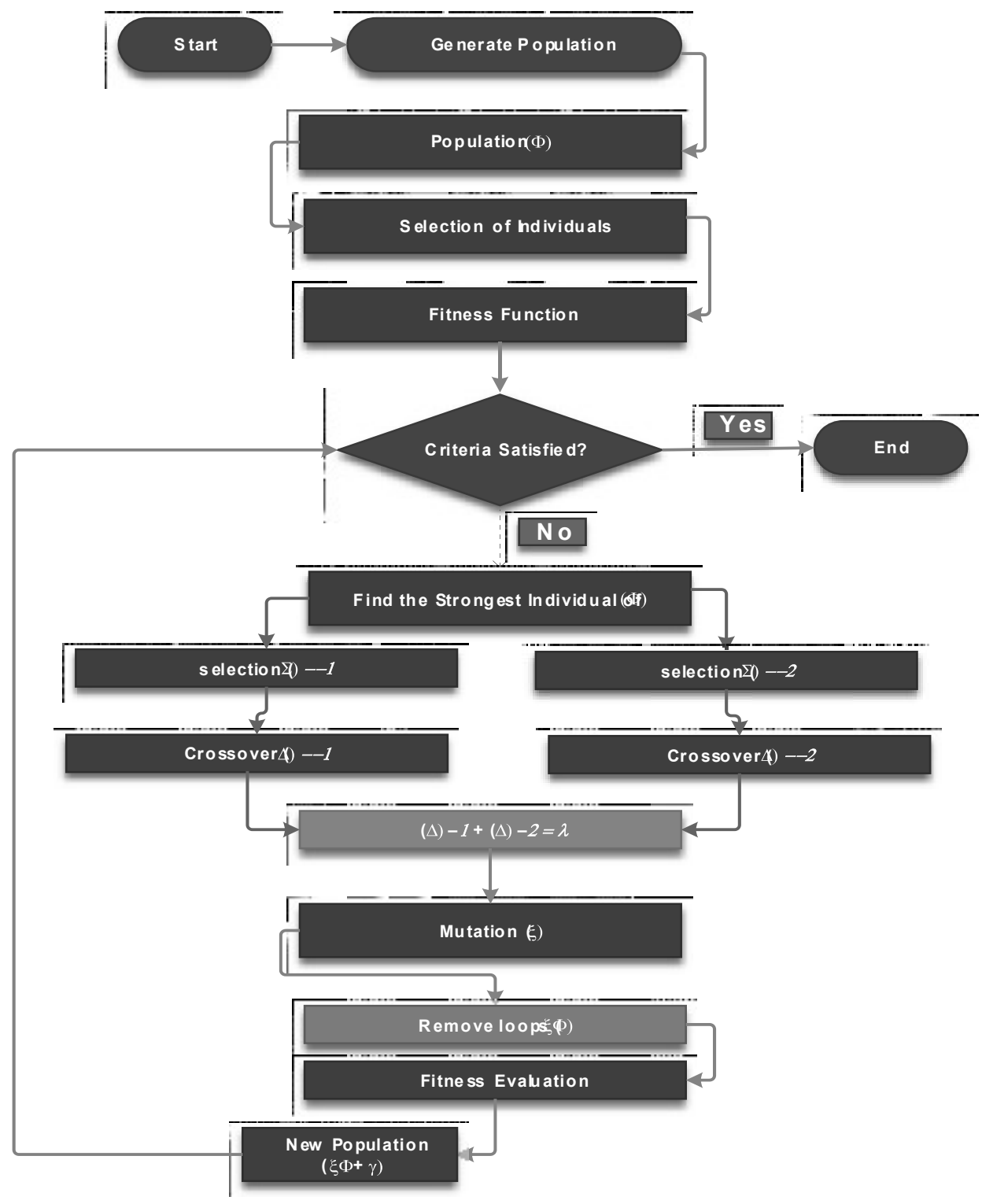

FIG. 2. GENETIC ALGORITHM OPERATORS

Mehran University Research Journal of Engineering \& Technology, Volume 38, No. 4, October, 2019 [p-ISSN: 0254-7821, e-ISSN: 2413-7219] 
individuals are called parent chromosomes which inherits an offspring chromosome. For the fit offspring individual the positions of the parents are randomly interchanged.

Mutation: In this operator the newly reproduced individuals are assumed to be mutated for the sake of better and strong genes through random selection as shown in Fig. 4. We apply mutation operator with the mutation probability of 0.7 . The operation applies like the crossover function but it further polish with fitness criteria for selection of better genes. Additionally, the operator avoids to select invalid with the help of fitness function.

Fitness Function: The FF (Fitness Function), is utilized for the fit individuals. Where each individual or chromosome undergoes through operation of selection on the basis of their specific residual energy. The fitness of robust chromosomes defines the healthiness of the selected individual among the rest of the individuals. The fit chromosomes are assumed to give birth more offsprings than other genes among the population (Equation (1)) [8].

$\mathbf{X}$ y

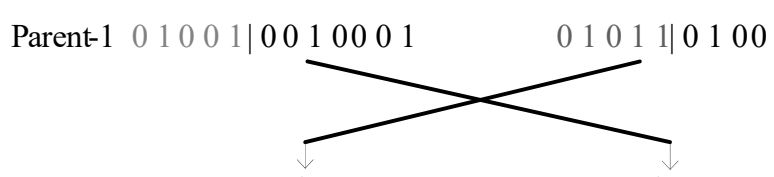

Parent-2 $0001000001 \mid 01110$

$01000 \mid 00101$

$\mathbf{X}$ y

Offspring $\quad 01011 \mid 0110$ \begin{tabular}{lllll|llllll}
0 & 1 & 0 & 0 & $1 \mid$ & 0 & 0 & 1 & 0 & 1
\end{tabular}

FIG. 3. CROSSOVER OF INDIVIDUALS .. 11001000... $\downarrow$ Mutation $\downarrow$ .. 00110001...

FIG. 4. MUTATION
$\mathrm{F}(\mathrm{x})=\frac{\mathrm{FF} \text { of Robust Individuals }}{\mathrm{FF} \text { of all Population }}$

By applying the above criteria we get fit gene if the value obtained 1 and further that gene is assumed to undergo the crossover function.

\subsection{Genetic Algorithms Optimization}

For WSNs, various research scholars endeavored for addressing the problems related to routing, energy efficient usage, and optimization using GA. A significant number of research has been focused upon resolution of the issue of energy. Optimization is one of the research for the increment of the lifespan of the WSN. Therefore, in the optimization scenario research demands more efforts for the sake of battery usage. The optimization schemes proposed by different scholars in $[8,13]$ using PSO (Particle Swarm Optimization) is a multi-dimensional technique. Another technique suggested by [14] for saving the energy with the help of GPS (Global Positioning System) for deployment of mobile sink at the distance of 1-hop from the SNs. The location of the mobile sink has been made known through GPS.

The research scholars of [15] proposed GA based optimization for deployment of SNs with respect to the area. The work of [16] focused upon optimization using multiple sinks. Another technique for localization SNs in a fully connected WSNs has been done utilizing GA. Similarly, the localization and placement of multiple sinks with the help of GA for LAN (Local Area Network) traffic has been addressed in [17]. The lifespan enhancement of WSNs using GA is proposed by [18-19].

\subsection{Simulation Results}

The simulations of the study are performed with the MATLAB R2016b. First, we employed four sinks stations at all four corners i.e. top right, top left, bottom 
right and bottom left, respectively. The network size number of SNs and other parameters as shown in Table 1, are assumed equally for both the proposed schemes [20].

Second, we have deployed 10 SNs and simulated the results. First, we deployed the sensors in a WSNs field. Third, the distance has been calculated of every SN from/to the nearest $\operatorname{sink}(\mathrm{s})$ with the help of GA, where we considered direct distance towards the sinks. Third, we evaluated the distance through multiple hops with the help of GA and then compared with [20].

When any node routes data towards the sink, before transmitting the data, it looks for the nearest and minimum distant neighbor nodes and routes the data when finds minimum distance between the neighbor nodes and nearest sink as shown in Fig. 5. The direct distance of the $\mathrm{SNs}$ form the respective sinks as illustrated in Table 2. The source node, i.e. $\mathrm{N}_{1}$, first calculate the distance from all sink and sends data towards Sink1 with minimum distance $\mathbf{6 5 . 7 9 5 1}$.

The SN N 2 route the data towards sink 4 with minimum distance of $\mathbf{2 0 . 6 1 5 5}$, and $\mathrm{N}_{6}$ routes through the minimum distant path of $\mathbf{4 6 . 8 6 1 5}$ towards the sink4. The $\mathrm{N}_{7}$ routes data directly towards the Sink1 with minimum distance $\mathbf{3 7 . 2 0 2 2}$ and $\mathrm{N}_{8}$ routes towards Sink1 with minimum distance 39.3954. $\mathrm{N}_{10}$ routes towards Sink1 with minimum distance $\mathbf{4 2 . 5 2 0 6}$.

The evaluation of the distance of each SN has been experimented for the sake of energy usage over the distance. The greater the distance among the SNs the greater amount of the energy will be used. To save the energy the distance must be least and optimum.

TABLE 1. SIMULATION PARAMETERS

\begin{tabular}{|c|c|}
\hline Network size & $100 \times 100 \mathrm{~m}^{2}$ \\
\hline No. of Nodes & 10 and 20 \\
\hline Initial energy of each node & $0.7 \mathrm{~J}$ \\
\hline Number of generations (rounds) & 4000 \\
\hline Energy dissipation & $50 \mathrm{~nJ} / \mathrm{bit}$ \\
\hline Energy Amplification $d_{-}<\mathrm{d}_{-} \mathrm{j}$ & $10 \mathrm{pJ} / \mathrm{bit} / \mathrm{m}$ \\
\hline Energy Amplification $d_{-}>\mathrm{d}_{-} \mathrm{j}$ & $0.0013 \mathrm{pJ} / \mathrm{bit} / \mathrm{m}$ \\
\hline
\end{tabular}

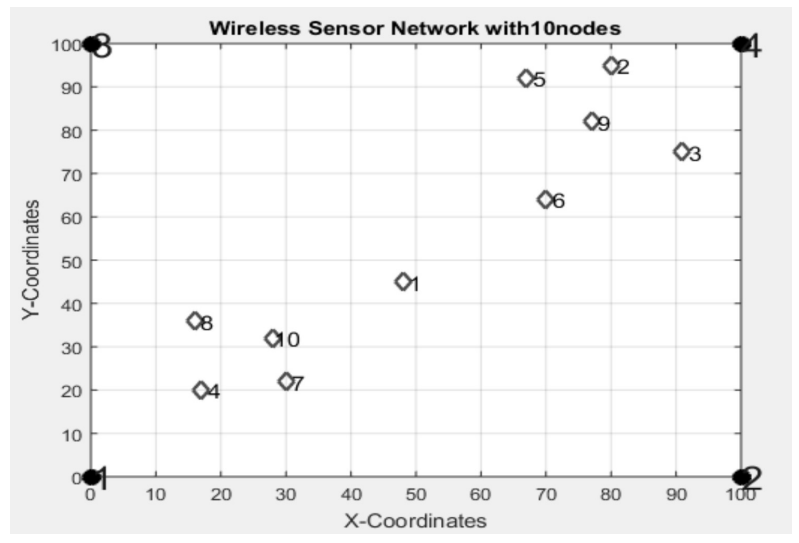

FIG. 5(a). THE SENSORS DEPLOYED RANDOMLY

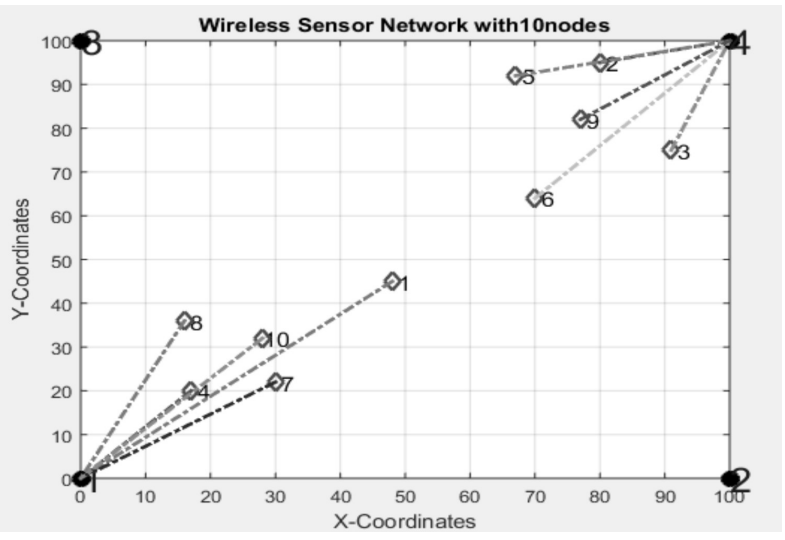

FIG. 5(b). DIRECT DISTANCE TOWARDS EACH SINK

Mehran University Research Journal of Engineering \& Technology, Volume 38, No. 4, October, 2019 [p-ISSN: 0254-7821, e-ISSN: 2413-7219] 
For the comparison simulation, we considered a WSNs with $10 \mathrm{SNs}$ deployed in an ad hoc manner as shown in Fig. 5(a) and the distance of all SNs from respective sinks as illustrated in Fig. 5(b). The direct distance of the SNs from the sinks helps in sending the data through minimum distant path. The path, furthermore, has been optimized through GA and data is routed through the best one from the available paths.

Notwithstanding, the direct distance is not the only option in WSNs for performing any routing scheme.
However, the distance between the SNs nearer to the

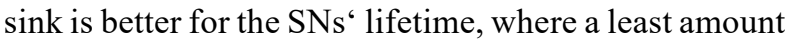
of energy may be used. But, if the path is not determined or scheduled the nearer SNs can transmit the data towards the farther sink rather than the nearest sink. The path and scheduling for routing the data is at top priority. The scheduling with the optimal paths to/from the SNs and sink achieves benefit of energy saving with the far and away. The sheer amplitude of energy is saved with the multiple hops communication paradigm.

TABLE 2. DIRECT DISTANCE OF SENSORS TOWARDS EACH SINK

\begin{tabular}{|c|c|c|c|c|}
\hline Sensor & Sink-1 & Sink-2 & Sink-3 & Sink-4 \\
\hline $\mathrm{N} 1$ & 93.9415 & 42.7200 & 104.0433 & 61.8466 \\
\hline $\mathrm{N} 2$ & 65.7951 & 68.7677 & 73 & 75.6902 \\
\hline N3 & 48.0104 & 81.8841 & 67.1193 & 94.3663 \\
\hline N4 & 124.1974 & 97.0824 & 80.1561 & 20.6155 \\
\hline N5 & 71.0211 & 112.4455 & 32.3109 & 92.9731 \\
\hline N6 & 33.2181 & 88.6760 & 72.8247 & 109.8337 \\
\hline N7 & 89.9889 & 116.1809 & 26.4197 & 78.0897 \\
\hline $\mathrm{N} 8$ & 61.2698 & 91.4002 & 52.4786 & 85.7555 \\
\hline N9 & 117.9237 & 75.5381 & 94.3716 & 26.5707 \\
\hline N10 & 80.9197 & 64.4049 & 78.4092 & 61.2209 \\
\hline N11 & 64.8459 & 51.0392 & 91.6788 & 82.4924 \\
\hline $\mathrm{N} 12$ & 48.4149 & 68.8767 & 79.6492 & 93.5094 \\
\hline N13 & 72.8011 & 41.2311 & 100.4988 & 80.6226 \\
\hline N14 & 28.6356 & 74.9667 & 91.7605 & 114.9783 \\
\hline N15 & 97.5756 & 41.4849 & 107.3359 & 61 \\
\hline N16 & 93.7443 & 91.5860 & 56.4624 & 52.8015 \\
\hline N17 & 36.8782 & 80.9938 & 75.8947 & 104.6900 \\
\hline N18 & 99.5691 & 94.4139 & 57.5674 & 48.1041 \\
\hline N19 & 77.6209 & 109.6586 & 32.0156 & 83.8153 \\
\hline $\mathrm{N} 20$ & 89.8999 & 43.3820 & 101.4002 & 63.8905 \\
\hline
\end{tabular}


The multi-hop scheme as shown in Fig. 6, illustrates the path length towards the nearest sinks, which consumes minimum effort for routing and results trivial energy consumption. The multiple hops reduce the distance from/to the nearest sinks through the relay SNs and results in optimized path to route the data. The data is routed through the optimal path towards the sink 1 with the $\mathbf{9 4 . 8 9 3 2}$ meters as illustrated in Fig. 6 (a-b) shows the total path distance towards $\operatorname{sink} 4$ is 93.329 meters.

We performed simulations by considering $10 \mathrm{SNs}$ deployed in a WSN as illustrated in Fig. 7, with Active nodes and compared to the standard algorithm [20], where $10 \mathrm{SNs}$ are deployed in a WSNs with $0.7 \mathrm{~J}$ of initial energy. The operation of the all of the active nodes have been assumed 4000 generations. In each generation/round the data is routed to the sinks. The number of active nodes remained operational or active and route the data towards the sinks. The results show better performance of the proposed algorithm contrary to the [20]. With equal number of rounds and same number of nodes the number of the dead nodes

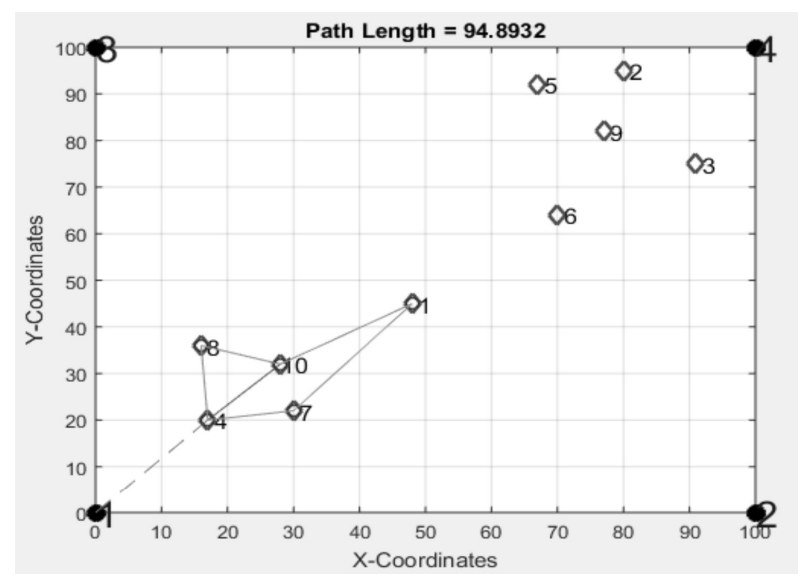

(a) SINK1 decreased as compared to the [20] as shown in Fig. 8, where the nodes die down slowly as compared to the [20]. The optimization through GA helps in reduction of the energy waste and resulted in improving the lifetime of the WSNs. The packet transmission of the proposed scheme advocates the better performance as compared to [20] as illustrated in Fig. 9, where the data packets received at the sinks are nearly 6000 while the data reception at the sinks in [20] remained nearly 2000 packets throughout the operation of the sensor nodes.

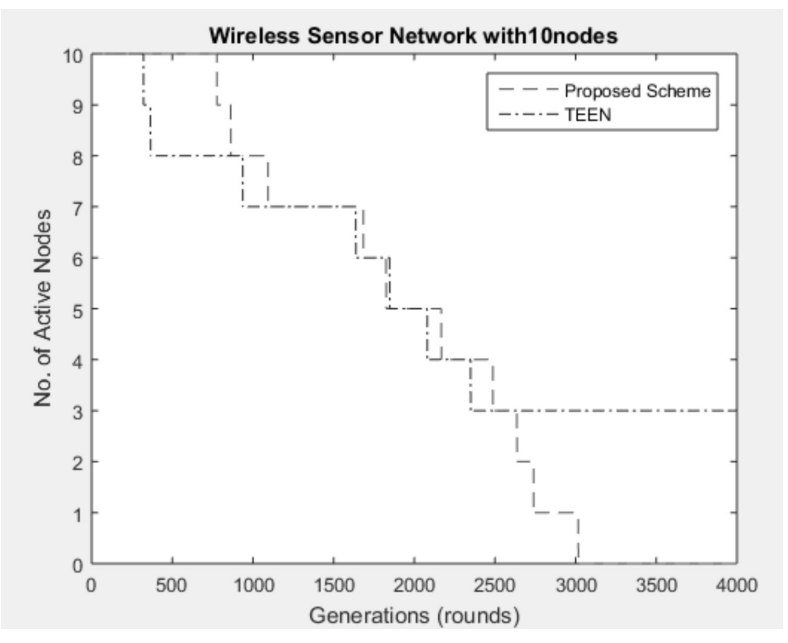

FIG. 7. COMPARISON OF ACTIVE NODES

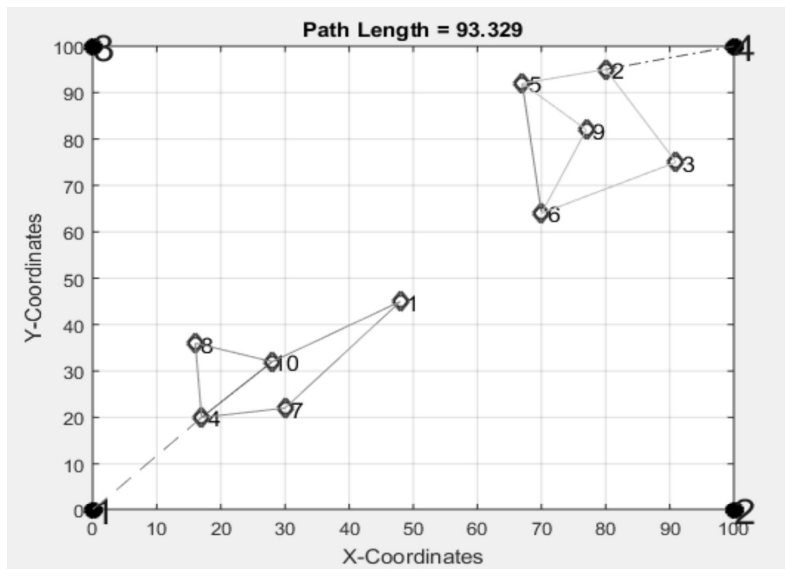

(b) SINK 4

FIG. 6. SENDING DATA 
After deployment of $10 \mathrm{SNs}$ the network has been considered a bit complex by employing 20 SNs. The study explores network operation and data routing schemes for the small scale WSNs to a complex one WSNs.

The WSNs with 20 SNs deployed in an ad hoc manner are shown in Fig. 10(a) and the distance of all SNs from respective sinks as illustrated in Fig. 10(b). The purpose of the distance calculation is route data through the least distant neighbor nodes to the nearest sink. We deployed and simulated the $20 \mathrm{SNs}$ in a WSNs,

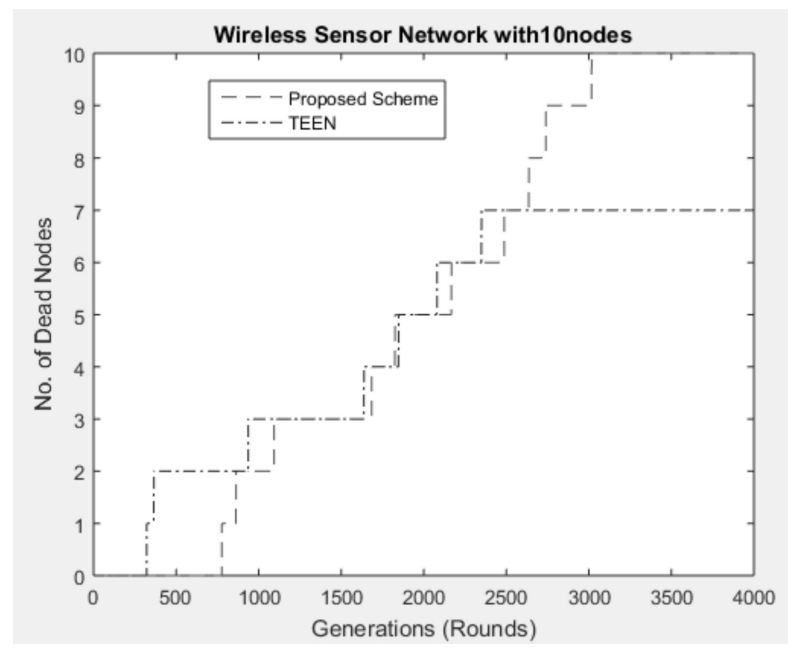

FIG. 8. COMPARISON OF DEAD NODES

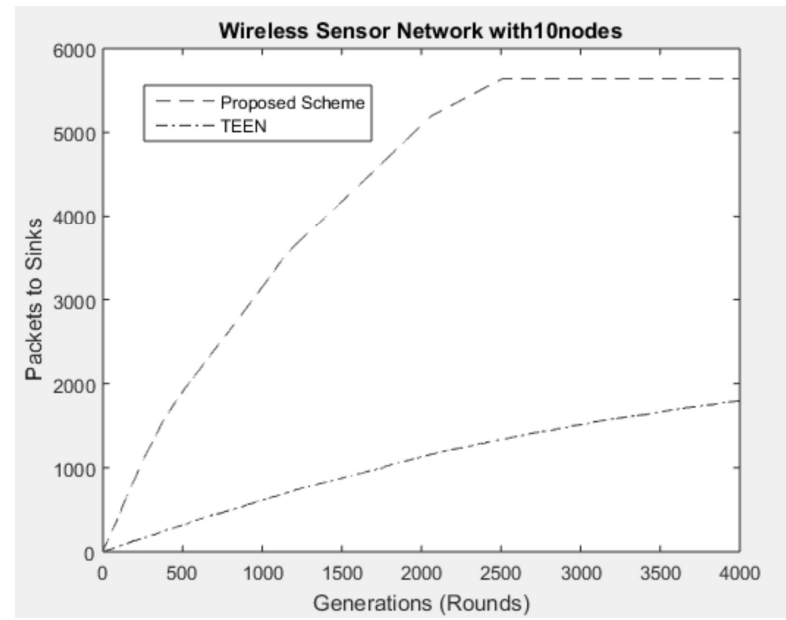

FIG. 9. COMPARISON OF PACKETS TO THE SINKS depicting the number of active nodes, we compared our proposed technique with the [20] as shown in Fig. 11. Simulation results show better performance of the proposed scheme as compared with [20]. With the proposed technique the number of Active SNs with respect to rounds are greater contrary to the [20] as illustrated in Fig. 12.

In Fig 11(a) sensors are routing through the multi-hop scheme and the optimum distance is $\mathbf{1 0 1 . 1 8 7 3}$ meters, while the sensors route the data through the minimum distance of 73.3833 meters as shown in Fig. 11(b-c)

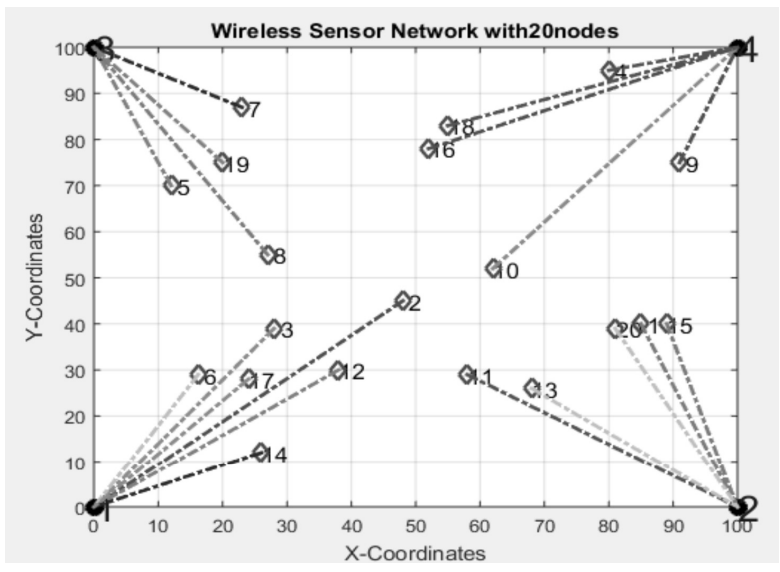

FIG. 10(a). THE SENSORS DEPLOYED RANDOMLY

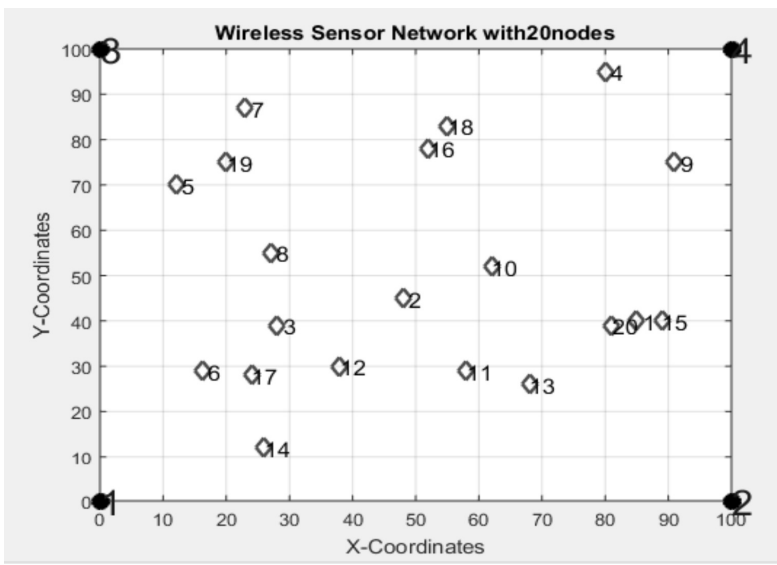

FIG. 10(b). DIRECT DISTANCE TOWARDS EACH SINK

Mehran University Research Journal of Engineering \& Technology, Volume 38, No. 4, October, 2019 [p-ISSN: 0254-7821, e-ISSN: 2413-7219] 
which shows the minimum path towards the sink 3 is 75.0206 while the SNs having minimum distance towards sink 4 over the optimal distance of $\mathbf{9 5 . 5 0 2 4}$ as illustrated in Fig. 11(d).

The path length towards each sink reduces the distance for the sake of routing the data towards the respective sink and helps in trivial energy consumption. The energy expenditure has been reduced through the multiple hops routing scheme.

The network performance in terms of dead nodes has been depicted in Fig. 13. The results shows the proposed scheme performs well with dead nodes per round as compared to [20]. The packet transmission of
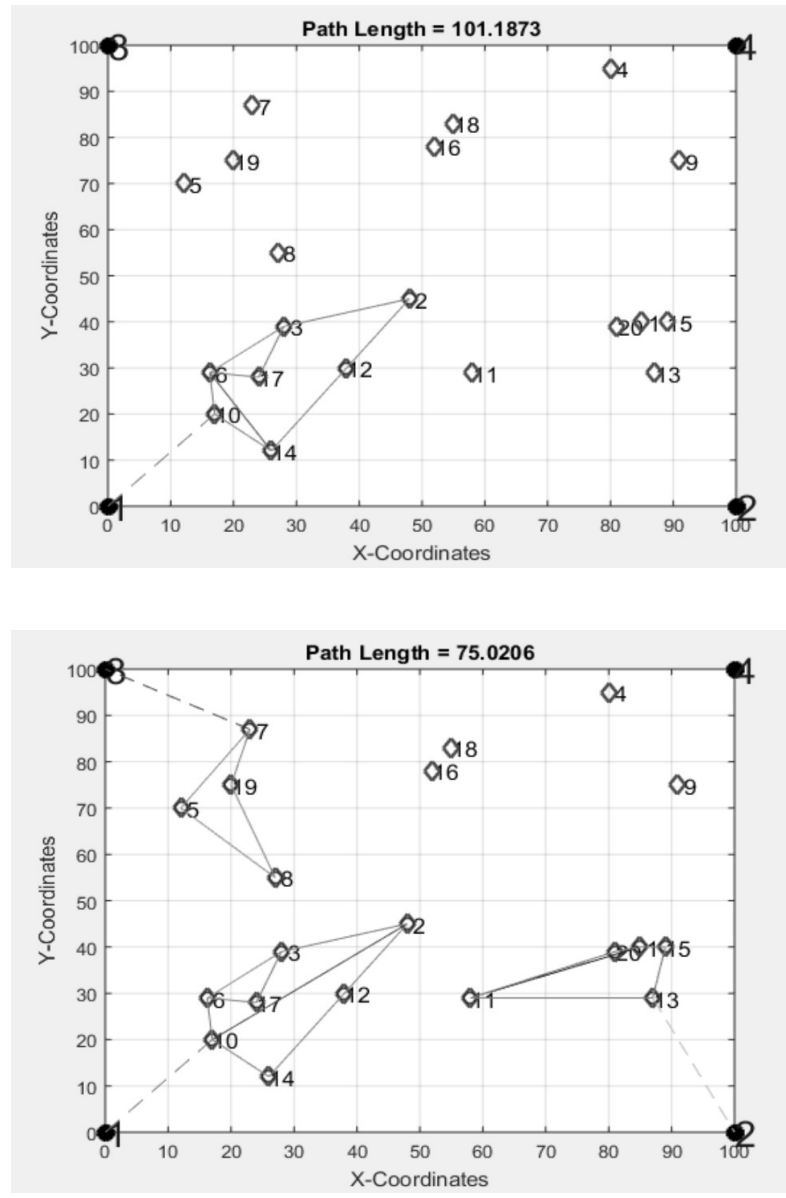

proposed scheme outperforms the [20] as shown in Fig. 14. The lifetime of the network increased and transmission scheme utilized efficiently.

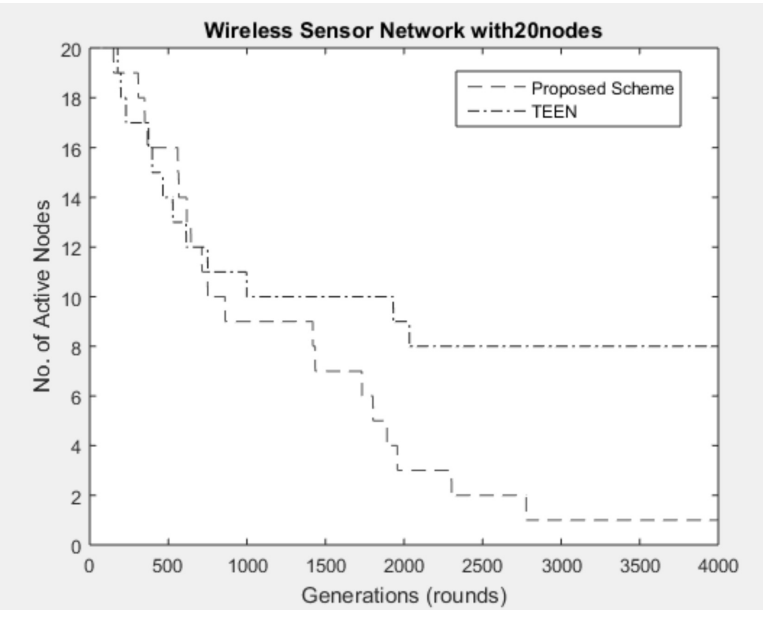

FIG. 12. COMPARISON OF ACTIVE NODES
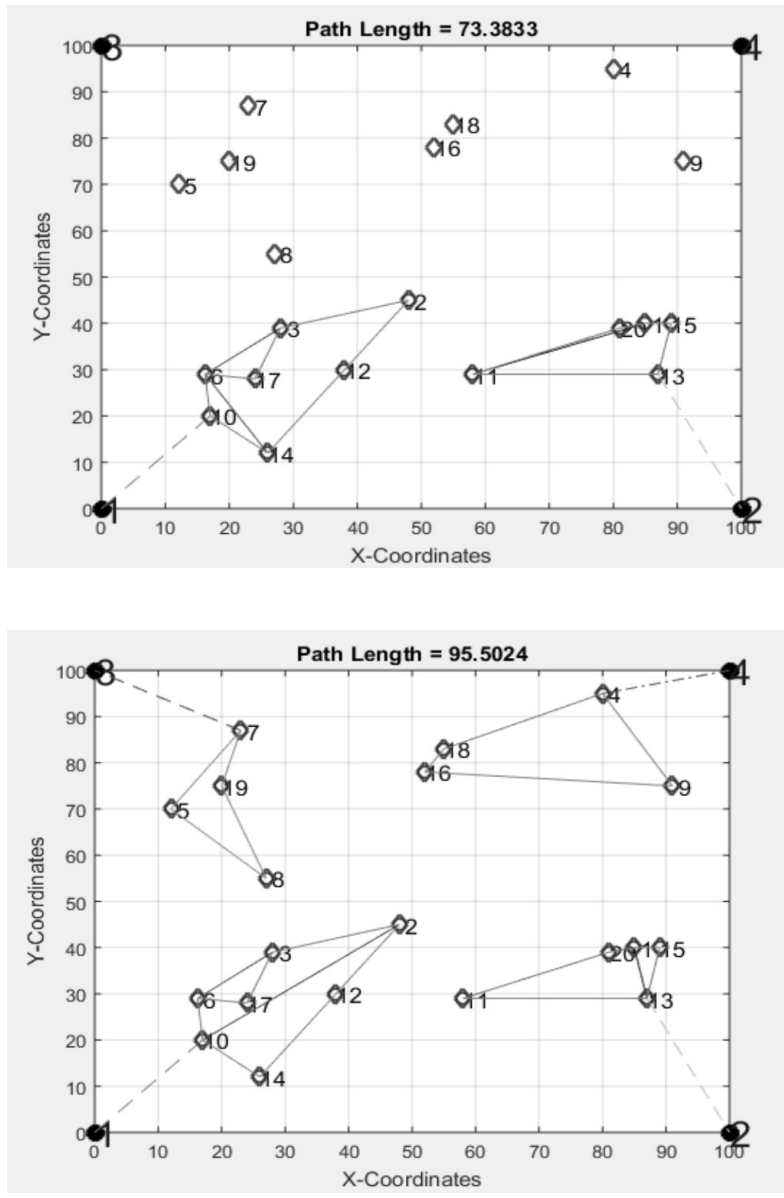

FIG. 11. THE SENSORS SEND DATA TOWARDS (a) SINK1, (b) SINK2, (c) SINK3 AND (d) SINK4 


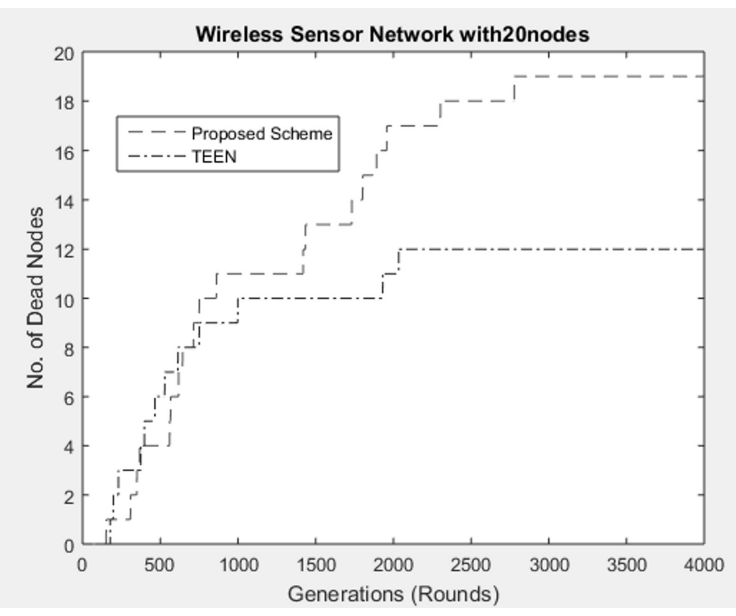

FIG. 13. COMPARISON OF DEAD NODES

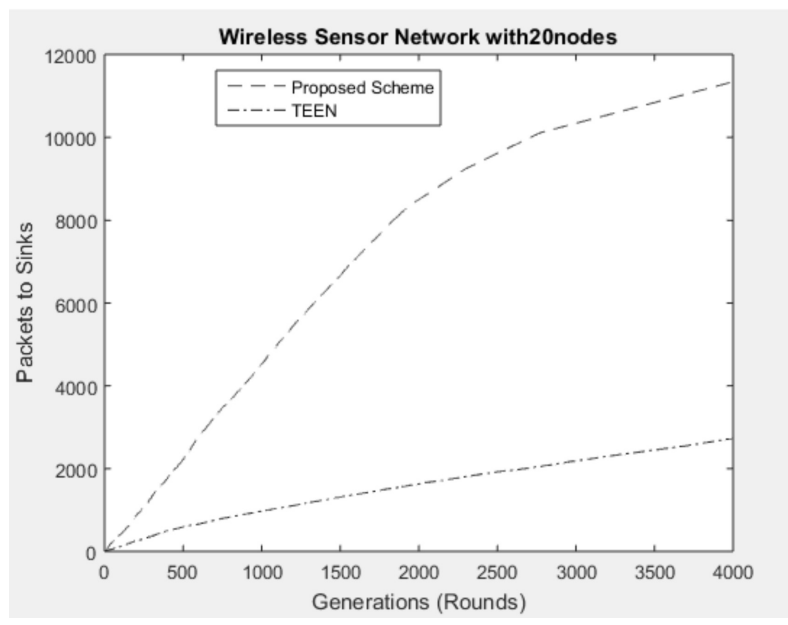

FIG. 14. COMPARISON OF PACKETS TO SINKS

\section{CONCLUSION}

In this paper a WSN scheduling technique for multiple sinks using GA has been presented, where four sinks are employed on four sides of the sensor field. The proposed scheme finds the optimized path using GA, during transmission of data from source node to the nearest sink. First, we run the GA for determination of routing paths, where a source $\mathrm{SN}$ find the possible number of optimal hops. Second, the hops or intermediate relay SNs are assumed to relay the data towards the sink, efficiently. The performance is experimented and evaluated using MATLAB R2016b.
The results of simulation comprise of 10 and 20 number of SNs, discretely. Additionally, the direct distance of each node is calculated and the distance through multiple hops from/to the nearest sink is also evaluated. The most optimized path selection is done with the help of GA from the available paths. The selected optimized route helps in transmitting the data towards the nearest possible sink by relaying hop(s). The path/route determination among all SNs from/to all sinks is done by GA. Then, GA determines the scheduling routing the data through optimal paths towards nearest sink. Thus, scheduling routing save the extra efforts for selecting route from multiple routes, in order to eschew routing over a long route and data consumption. Therefore, the network performance and lifetime is enhanced significantly through proposed scheme. The proposed scheme is proved an efficient method to save both energy and distance for the sake of network longevity and optimal and precise data delivery by multiple hops. The results of the proposed scheme are very interesting and the lifetime of the WSNs have been considerably enhanced as comparing with standard TEEN algorithm. The study bears more successful results when the network size goes small to complex. Thus, the network lifetime increased by the proposed scheduling scheme.

\section{FUTURE DIRECTIONS}

The research areas and scopes for enhancement of lifespan of WSNs have been flourished using multiple hops routing scheme. Though there is still challenging aspects are remaining. A robust routing protocol is needed which saves the energy without fail. A routing technique with more complex network using homogeneous sensor nodes will be the future work.

\section{ACKNOWLEDGEMENT}

The authors are indebted to the Institute of Information and Communication Technology, University of Sindh, Jamshoro, Pakistan, for providing computing facilities in support of the research study. The university management has provided fullsupport in the completion of the research.

Mehran University Research Journal of Engineering \& Technology, Volume 38, No. 4, October, 2019 [p-ISSN: 0254-7821, e-ISSN: 2413-7219] 


\section{REFERENCES}

[1] Solangi, S.A., Hakro, D.N., Lashari, I.A., Khoumbati, K.-R., Bhutto, Z.A., and Hameed, M., "Genetic Algorithm Applications in Wireless Sensor Networks (WSN): A Review," International Journal of Management Sciences and Business Research, Volume 1, No. 4, pp. 152-166, 2017.

[2] Tahir, M., and Qureshi, R., "A Survey of Energy Conservation Mechanisms for Dynamic Cluster Based Wireless Sensor Networks", Mehran University Research Journal of Engineering \& Technology, Volume 37, No. 2, pp.279-296, Jamshoro, Pakistan, April, 2018.

[3] Baranidharan, B., and Santhi, B., "GAECH: Genetic Algorithm Based Energy Efficient Clustering Hierarchy in Wireless Sensor Networks", Journal of Sensors, pp. 1-8, 2015.

[4] Ismat, N., and Qureshi, R., "Energy and Round Time Estimation Method for Mobile Wireless Sensor Networks", Mehran University Research Journal of Engineering \& Technology, Volume 37, No. 1, pp. 105-118, Jamshoro, Pakistan, January, 2018.

[5] Jha, S.K., and Eyong, E.M., "An Energy Optimization in Wireless Sensor Networks by Using Genetic Algorithm", Telecommunication Systems, Volume 67, No. 1, pp. 113-121, 2018.

[6] Sasi, S.B., andSivanandam, N., "A Survey on Cryptography Using Optimization Algorithms in WSNs", Indian Journal of Science and Technology, Volume 8, No. 3, pp. 216-221, 2015.

[7] Al-Shalabi, M., Anbar, M., and Wan, T.C., "Genetic Algorithm Based Protocols to Select Cluster Heads and Find Multi-Hop Path in Wireless Sensor Networks", MATEC Web of Conferences, pp. 1-7, 2018.

[8] Khan, F.H., Shams, R., Umair, M., and Waseem, M., "Deployment of Sensors to Optimize the Network Coverage Using Genetic Algorithm", Sir Syed University Research Journal of Engineering \& Technology, Volume 2, No. 1, pp. 8-11, Karachi, Pakistan, 2012.

[9] Gupta, T., "Implementation of an Optimization Technique: Genetic Algorithm", International Journal of Advanced Research in Computer Engineering \& Technology, Volume 4, No. 12, pp. 4359-4364, 2015.

[10] Memon, M.A., Memon, S., Bhatti, Z., Khowaja, S., and Baloch, B., "Autonomous Robot Path Planning Using Particle Swarm Optimization in Static and Obstacle Environment", Sindh University Research Journal (Science Series), Volume 47, No.4, pp. 653-658, Jamshoro, Pakistan, 2015.
[11] Wei, G., and Xie, X., "Research of Using Genetic Algorithm of Improvement to Compute the Most ShortPath", 3rd International Conference onAntiCounterfeiting, Security, and Identification in Communication, pp. 516-519, 2009.

[12] Wang, F., Wang, C., Wang, Z., and Zhang, X.Y., “A Hybrid Algorithm of GA+ Simplex Method in the WSN Localization", International Journal of Distributed Sensor Networks, Volume 11, No. 7, pp. 1-9, 2015.

[13] Latiff, N.A., Tsimenidis, C.C., and Sharif, B.S., "EnergyAware Clustering for Wireless Sensor Networks Using Particle Swarm Optimization", IEEE 18th International Symposium on Personal, Indoor and Mobile Radio Communications, pp. 1-5, 2007.

[14] Chen, Y., Wang, Z., Ren, T., and Lv, H., "Lifetime Optimization Algorithm with Mobile Sink Nodes for Wireless Sensor Networks Based on Location Information", International Journal of Distributed Sensor Networks, Volume 11, No. 8, 2015.

[15] Kalayci, T.E., and Uður, A., "Genetic Algorithm-Based Sensor Deployment with Area Priority", Cybernetics and Systems, Volume 42, No. 8, pp. 605-620. 2011.

[16] Norouzi, A., and Zaim, A.H., "Genetic Algorithm Application in Optimization of Wireless Sensor Networks", ScientificWorldJournal, No. 2, pp. 1-15, 2014.

[17] Poe, W.Y., and Schmitt, J.B., "Placing Multiple Sinks in Time-Sensitive Wireless Sensor Networks Using a Genetic Algorithm",14th GI/ITG Conference on Measuring, Modelling and Evaluation of Computer and Communication Systems, pp. 1-15, 2008.

[18] Singh, M.K., and Nagarathna, P., "Improvement in Life Span of WSN Using Genetic Algorithm with New Fitness Function" Proceedings of IEEE International Conference on Electronics Computer Technology, pp. 1-23, 2012.

[19] Bhulania, P., Gaur, N., and Federick, K.P., "Improvement of Lifetime Duty Cycle Using Genetic Algorithm and Network Coding in Wireless Sensor Networks",6th International Conference on Cloud System and Big Data Engineering (Confluence), pp. 611-618, 2016.

[20] Manjeshwar, A., and Agrawal, D.P., “TEEN: ARouting Protocol for Enhanced Efficiency in Wireless Sensor Networks", IEEE Computer Society, pp. 1-7, 2001. 